\title{
Assessment of Virulence Factors and Antifungal Susceptibility of Candida Species Isolated from Catheter Associated Urinary Tract Infections
}

\section{Manal Gomaa Ismail ${ }^{1}$ MD; Naglaa Farouk Abd El Haliem ${ }^{1}$ MD; Enas Kamal Abo-El-magd ${ }^{*}$ MD}

\author{
*Corresponding Author: \\ Enas Kamal Abo El-maged \\ enasaboelmagd@yahoo.com
}

Received for publication July 10, 2020; Accepted September 30, 2020; Published online September 30, 2020.

Copyright 2020 The Authors published by Al-Azhar University, Faculty of Medicine, Cairo, Egypt. All rights reserved. This an openaccess article distributed under the legal terms, where it is permissible to download and share the work provided it is properly cited. The work cannot be changed in anyway or used commercially.

doi: 10.21608/aimj.2020.35322.1278

${ }^{1}$ Microbiology and Immunology Department, Faculty of Medicine For Girls, Al-Azhar University, Cairo, Egypt.

Disclosure: The authors have no financial interest to declare in relation to the content of this article. The Article Processing Charge was paid for by the authors.

Authorship: All authors have a substantial contribution to the article.

\begin{abstract}
Background: Catheter associated urinary tract infection (CAUTI) is a crucial attribute to virulence of organisms. Emergency of non albicans Candida (NAC) species become common due to several virulence factors, which play an important role in pathogenicity and their resistance to treatment.

Aim of the work: To investigate different virulence factors of Candida spp. isolated from CAUTI patients and evaluate their antifungal susceptibility.

Patients and Methods: Urine samples collected from 219 indwelling catheters of ICU patients. Candida isolates identified using Germ tube test, Chrom ID agar, Corn meal tween agar, VITEK 2 system and Integral System Yeast Plus (ISYP) used to detect antifungal susceptibility. Enzymatic, haemolytic activity and biofilm formation assessed by standard methods.

Results: The incidence of candiduria was 50/219 (22.8\%). Candida tropicalis was predominant one (54.0\%) followed by C. albicans (26.0\%) and C. glabrata (20.0\%). Females gender (64.0\%), Diabetes $(50.0 \%)$ associated with positive candiduria, while antibiotic therapy was predominant risk factor $(78.0 \%)$. C. albicans have highest enzymatic activity for phospholipase (84.6\%), proteinase (84.6\%) and haemolytic activity (100\%), while C. tropicalis have highest biofilm formation (100\%). NAC spp. showed higher rates of resistance to azole drugs (Clotrimazole 77.6\%, Miconazole 70.3\%, Econazole 63.2\%, Nystatine 44.3\%), while C. albicans didn't show any resistance.

Conclusion: Candiduria is prevalent among catheterized patients with shift toward NAC spp. causing nosocomial UTI. A strong relationship between host risk factors (old age, antibiotic use, catheterization, female sex, ICU stay, diabetes) and expression of various virulence factors of Candida spp. causing candiduria and resistance to antifungal drugs.
\end{abstract}

Keywords: Candiduria; virulence factors; antifungal resistance.

Candiduria, firulence

\section{INTRODUCTION}

Urinary tract infection (UTI) is one of the most common infections in clinical life. They can be caused by a range of pathogens such as bacteria, fungi, parasites, and viruses ${ }^{1}$. However, among the fungal agents, and the opportunistic fungal infections involving urinary system, Candida species are the most common causes of $\mathrm{UTI}^{2}$.

Candida spp. can be colonized in the lower or upper urinary tract system and create pyelonephritis, cystitis, urethritis and prostatitis in men ${ }^{3}$. They might even appear in the upper urinary tract from the bloodstream or raise the urinary tract from a focus of Candida colonization at near the urethra ${ }^{4}$. Although C. albicans is the most commonly isolated species in candiduria, the incidence of non albicans species is ever increasing ${ }^{5}$.
Predisposing conditions such as extensive use of broad-spectrum antibiotics, diabetes mellitus, urinary stasis, renal transplantation, and hospitalization can increase the risk of candiduria ${ }^{6}$. Also, urinary catheters are a crucial attribute to virulence as they allow the yeast to attach to body sites and commence proliferation and colonization. Candida species have several virulence factors that facilitate proliferation; they may result in adhesion to the epithelium and invasion of the host tissue.

The extracellular hydrolytic enzymes including secreted aspartyl proteinase and phospholipases ${ }^{7}$. Furthermore, the survival and ability of Candida albicans to establish infections within humans are mainly related to its ability to produce elemental iron through haemolysin production. Moreover, it has been reported that biofilm formation also plays an essential role in the pathogenicity of Candida 
species $^{8}$. The aim of this work is to investigate various virulence factors as phospholipase, proteinase and haemolytic activities in Candida species isolated from patients with catheter associated urinary tract infection, assess their ability to biofilm formation and evaluate the antifungal susceptibility pattern of the isolated Candida species.

\section{PATIENTS AND METHODS}

Study population: A total of 219 patients admitted to ICU of Tanta University Hospital and undergoing urethral catheterization for more than seven days with negative urine cultures at the time of admission were investigated. They were divided into 125 female and 94 male patients. Their age ranged from 50 -90 years old with mean \pm SD $(62.45 \pm 7.93)$. Verbal informed consent was taken from patients to participate in the study.

Inclusion Criteria: Hospitalized ICU patients on continuous catheterization for more than 7 days with negative urine cultures at the time of admission

Exclusion Criteria: Patients with history of UTI prior to admission and patients with duration of catheterization less than 7days.

Ethical approval: written approval was obtained from the Ethical Research Board (ERB) of the Faculty of Medicine for girls, Al-Azhar University, Cairo, Egypt.

Collection of samples: After obtaining verbal informed consent and changing the catheter about 10 $\mathrm{ml}$ of urine sample was collected immediately from each patient using a sterile syringe through the sample port of the indwelling catheter under complete aseptic precautions. Each sample was transferred into two sterile screw capped labelled falcon tube. The tubes containing the samples were transferred on ice directly to our microbiology laboratory where processing of samples was carried out without any delay.

Samples Processing: Ten microliters from each urine sample were plated on Sabouraud's dextrose agar (SDA) plate supplemented with gentamicin $(50 \mathrm{mg} / \mathrm{L})$ and chloramphenicol $(0.05 \mathrm{gms} / \mathrm{L})$, blood agar and CLED medium using calibrated loop. Plates were incubated aerobically at $37^{\circ} \mathrm{C}$ for 24-48 hours ${ }^{9}$. Presumptive identification of Candida isolates were done morphologically as white to creamy, round, soft and smooth wrinkled colonies with characteristic yeast odor. Microscopic examination of Gram stained film prepared from suggestive colonies showed Grampositive budding yeast cells with or without pseudohyphae.

Colony counts (CFU) were done for all positive urine samples. All Candida isolates with CFU $\geq$ $10^{3}$ were purified by a repeated streaking method on SDA to obtain pure discrete colonies, that stored in $10 \%$ glycerol (Sigma-Aldrich, South Africa) at $80^{\circ} \mathrm{C}$ until further investigation.

Species Identification: Isolated yeast colonies were speciated phenotypically by germ tube test, chromogenic medium \{Chrom ID Candida Agar (CAN2)\}, chlamydospore formation on corn meal tween 80, and sugar assimilation by Integral System Yeast Plus Commercial Kit (Liofilchem, Ref.71822 - ITALY). and confirmed by Vitek 2 system. Standard strains of C. albicans (ATCC, 10231), C. tropicalis (ATCC750) and C. glabrata (ATCC, 2001) were used as controls in all investigation.

1.Germ tube test: Fifty microliters of each yeast cell suspension with a turbidity of $1.5 \times 103$ cells/ml equivalent to $0.5 \mathrm{McF}$ arland standard was inoculated in $2 \mathrm{ml}$ Eppendorf tubes containing aliquots of bovine serum albumin and incubated aerobically at $37^{\circ} \mathrm{C}$ for three hours. Then, centrifugation of the inoculated tubes was done. A wet mount film was prepared and examined under the microscope to look for the presence of germ tube. The germ tube appeared as short lateral hyphen filament (Figure 1). There is no point of constriction at the origin of the germ tube and is classically described as hand mirror appearance ${ }^{11}$. C. albicans and $\mathrm{C}$ dubliniensis produce germ tubes. C. tropicalis may produce early pesudohyphae that are constricted at the point of origin with the yeast cell in contrast to germ tube (Figure 1). Other candida species give no hyphae or germ tube.

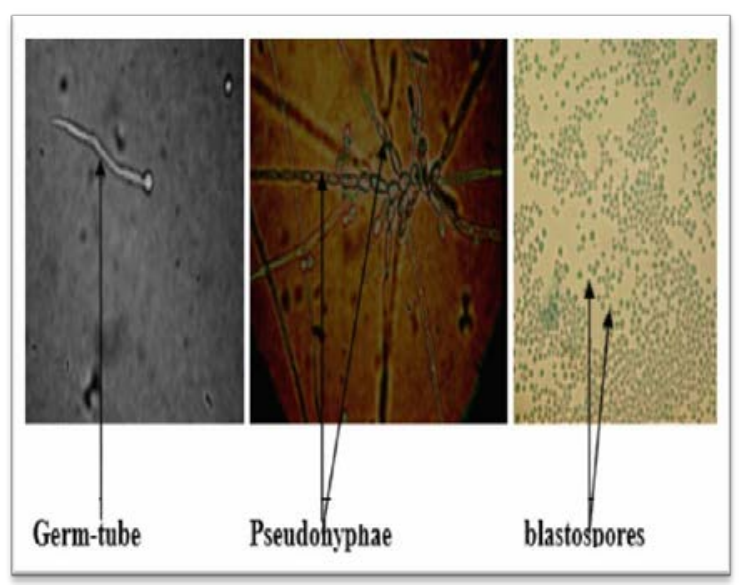

Fig 1 : Germ tube test of isolated candida species

2.Chromogenic medium: Fresh Candida isolates were cultured on ChromID Candida Agar \{(CAN2), bioMerieux $\}$ and incubated for 48 hours at $37^{\circ} \mathrm{C}$. Candida albicans appear as blue colonies, C.tropicalis appear pink while other Candida spp. appear as creamy white colonies (Figure 2). 


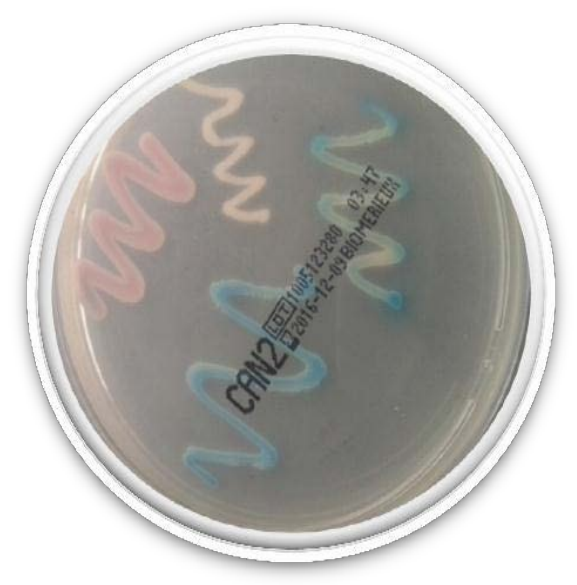

Fig 2: Colonies of Candida on Chrom ID agar (CAN2)

3. Chlamydospore formation: All Candida isolates were tested to produce chlamydospores on corn meal agar supplemented with $1 \%(\mathrm{v} / \mathrm{v})$

Tween 80(CMA-Tween 80)12. Positive testing isolates had feathery or spidery outgrowths with spores. Negative culture plates showed no visible sign of morphological change upon examination, but rather maintained their typical blastopore stage (Figure 3).

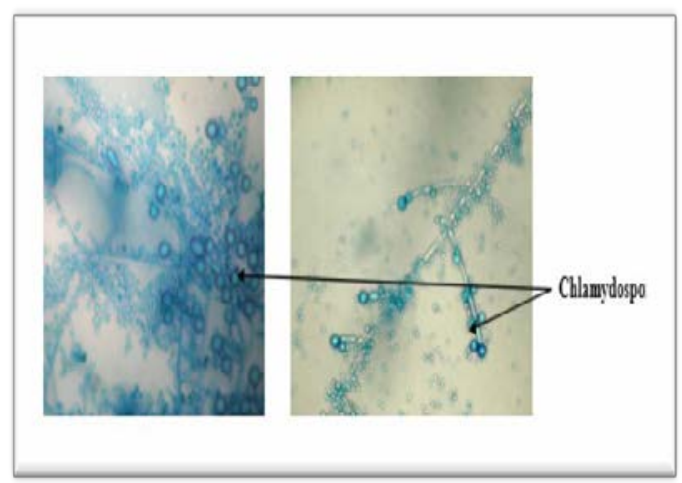

Fig 3 : Chlamydospores formation on CMA-Tween 80 agar

Sugar assimilation: by Integral System Yeast Plus Commercial Kit (Liofilchem, Ref.71822 - ITALY) according to the manufacturer's instructions. The results were comparable with the standard ATCC strains. The specific pattern of sugar assimilation was observed, then a specific code was calculated, and the species name was known from the codebook. All strains of C. albican and C. tropicalis were identified through numerical code formation and color change in chromogenic well. Some C. glabrata isolates were misidentified by this system as it gives variable color change (Figure 4), and confirmed by Vitek 2 system.

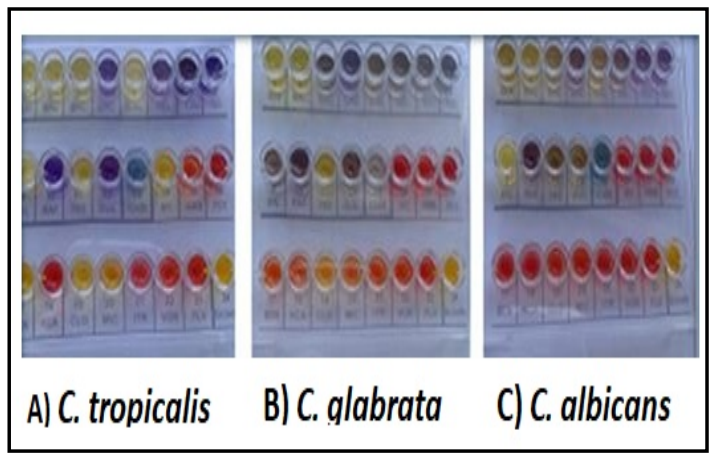

Fig 4: Sugar assimilation profile of isolated Candida species by Integral System Yeast Plus

(ISYP)

Antifungal Susceptibility Test :

The antifungal susceptibility profiles of all Candida isolates were determined against a panel of antimycotics using the Integral System Yeast Plus (ISYP) (Liofilchem, Ref. 71822 -ITALY) according to the manufacturer's instructions. Drug sensitivity was evaluated according to growth or inhibition of yeasts in media containing the antimycotic and a growth indicator.

Detection of Virulence Factors Candida Species

Extracellular Phospholipase Activity: Candida species were investigated for extracellular phospholipase using the egg yolk agar plate method. Briefly, $10 \mu \mathrm{L}$ of each standardized cell suspension carefully spotted on two plates of egg yolk agar medium and incubated $37^{\circ} \mathrm{C}$ for 48 hours (Figure, 5A). Phospholipase activity (Pz) was measured and interpreted for each isolate as previously described by Price etal, (1982)13. Reference strains of C. albicans (ATCC, 10231), C tropicalis ATCC750 and C. glabrata (ATCC, 2001) were used as controls.

Aspartyl proteinase activity: using Bovine serum albumin (BSA) agar plate's method (Figure, 5B. Briefly by adding $10 \mu \mathrm{L}$ of each standardized cell suspension to a sterile paper disk, which was applied to the surface of BSA medium that was incubated at $37{ }^{\circ} \mathrm{C}$ for five days. Then, the plates were fixed with $20 \%$ trichloroacetic acid (TCA) and 
stained with 1\% Amido Black dye 14. Enzyme activities were scored according to the criteria by Patil et al (2014)15. Reference strains of C. albicans (ATCC, 10231), C tropicalis ATCC750 and C. glabrata (ATCC, 2001) were used as controls.

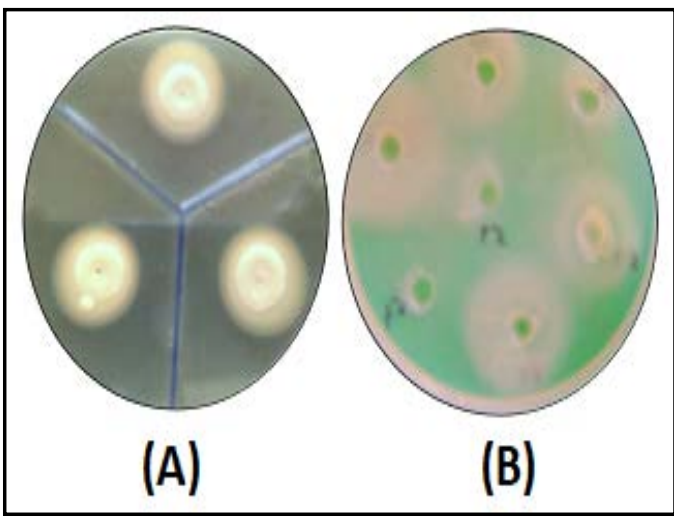

Fig 5: Phospholipase (A) and Aspartyl proteinase (B) activities by Candida species

Hemolysin activity: Ten microliters of standardized cell suspension of each isolates was spot inoculated on an enriched sheep blood SDA agar medium in triplicate on three separate occasions. Plates were incubated at $37^{\circ} \mathrm{C}$ for 48 hours. C. albicans (ATCC 10231) and Streptococcus pyogenes (group A) were used as positive controls for $\beta$ and $\alpha$ hemolysis, respectively. The presence of a distinctive translucent area around the inoculum indicated positive hemolytic activity. Hemolytic index (Hi) equal the ratio of the diameter of the colony to total diameter of the colony plus the translucent area16.

Biofilm formation: Biofilm production was determined by two methods:-

Tube method: A loopful from overnight culture of SDA plate for each isolate was inoculated into a polystyrene Falcon conical tube with a screw cap, containing $10 \mathrm{ml}$ of Sabouraud-dextrose broth with glucose $8 \%$ and incubated for $24 \mathrm{~h}$ at $37^{\circ} \mathrm{C}$. Then the broth was gently aspirated followed by washing of the tubes with distilled water and staining with $0.1 \%$ crystal violet. Excess stain was washed with deionized water. Tubes were dried inverted and then examined for the presence of an adherent layer (Figure, 6). Biofilm production was scored as negative $(-)$, weak $(+)$, moderate $(++)$ or strong $(+++) 17$.

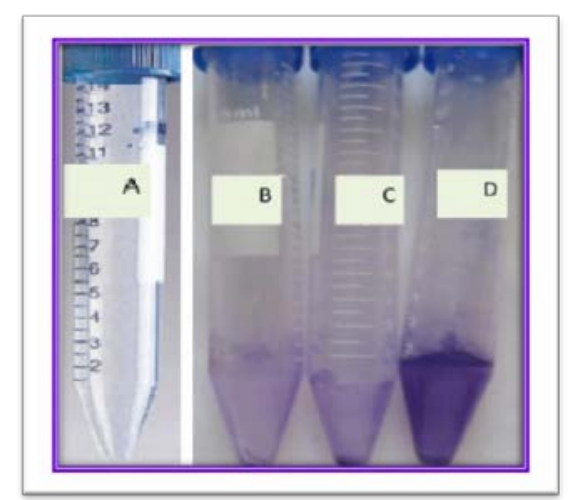

Fig 6: Biofilm detection by tube method stained with crystal violet.

A (negative), B (+), C (++), D (+++)

Congo red Method: Congo red agar medium plates were inoculated with Candida isolates incubated at $37^{\circ} \mathrm{C}$ for $24-48$ hours aerobically18. Positive result was indicated by red colonies, and negative results were indicated by white or light pink- colored colonies (Figure 7).

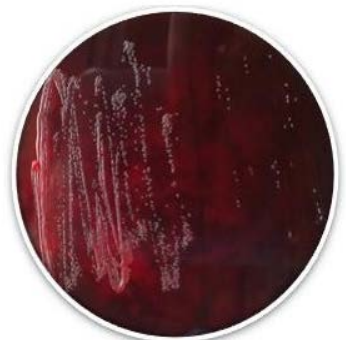

positive biofilm producer

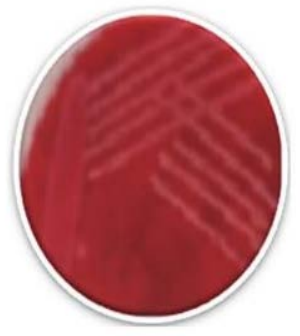

Negative biofilm producer
Fig 7: Biofilm detection by Congo red method. The left plate shows positive biofilm producer (red colonies), While, the right plate shows negative (pale pink colonies).

\section{VITEK 2 System:}

The ten isolates suspected to be C. glabrata were tested by VITEK 2 system according to the manufacturer instructions to confirm their identification.

Statistical Analysis: Statistical analysis was conducted, using the mean, standard Deviation, unpaired student t-test, and chi-square tests by SPSS V20. 


\section{RESULTS}

Two hundred and nineteen patients were investigated for candiduria; $125 / 219$ (57.1\%) patients were female and 94/219 (42.9\%) were males. The patients' ages ranged from $50-90$ years with mean \pm SD (62.45 \pm 7.93$)$. Table (1) shows the frequency of different predisposing risk factors found among the studied patients.

\begin{tabular}{|l|c|c|}
\hline \multicolumn{1}{|c|}{ Risk factors } & No.(219) & (\%) \\
\hline Urinary catheterization & 219 & 100.0 \\
Immunosuppressive therapy & 22 & 10.0 \\
Antibiotic therapy & 186 & 84.9 \\
Sepsis & 12 & 5.5 \\
Diabetes mellitus & 84 & 38.3 \\
Liver disease & 73 & 33.3 \\
Chronic kidney disease & 28 & 12.8 \\
Malignancy & 22 & 10.0 \\
\hline
\end{tabular}

Table 1: Frequencies of predisposing factors among the studied patients.

Incidence of candida isolation: Fifty out of two hundred and nineteen (22.8\%) urine samples were positive for Candida spp., while bacterial growth was detected in 132/219 (60.3\%) and 37/219 (16.9\%) was negative growth, \{Figure 8, (A)\}. Candida tropicalis was the most predominant one 27/50 (54.0\%) followed by C. albicans 13/50 (26.0\%), then C. glabrata 10/50 (20.0\%), \{Figure 8. (B)\}.

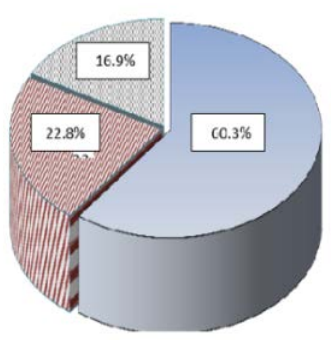

(A)

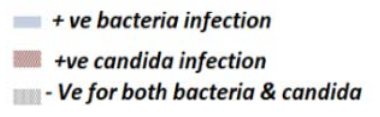

Fig 8: Frequency of Candida infection (A) and distribution of different candida species (B) among tested patients.

Speciation of Candida isolates

(1) Germ tube test: C. albican form germ tube, while C. tropicalis form pseudohyphae, but other isolates didn't form germ tube.

(2) Chrom ID Agar: C. albican produce blue colonies and C. tropicalis produce pink colonies while all other Candida species produce white colonies, Figure (2).

(3) Chlamydospores formation on CMA-Tween 80 agar: C. albican produce chlamydospore, C. tropicalis produce pseudohyphae, with clusters of blastoconidiae at the center, while C. glabrata didn't produce hyphae or phseudohyphae, Figure (3).

(4) Sugar assimilation: all strains of C. albican and C. tropicalis were identified through numerical code formation and color change in chromogenic well. The specific pattern of sugar assimilation was observed, then a specific code was calculated, and the species name was known from the codebook. The results were comparable with the standard ATCC strains. Some C. glabrata isolates misidentification by this system Figure (4). and confirmed by Vitek 2 system.

Virulence factors of Candida Species:

Phospholipase activity: production of phospholipase was higher in C. albicans than that of NAC species as $84.6 \%(11 / 13)$ of the C. albicans isolates had strong phospholipase activity (+++), $15.4 \%(2 / 13)$ had moderate enzymatic activity $(++)$. While, $44.4 \%$ $(12 / 27)$ of C. tropicalis had no phospholipase (-) and $55.6 \%(15 / 27)$ had weak enzymatic activity $(+)$. However, C. glabrata isolates had no enzymatic activity at all. The difference was statistically highly significant $(\mathrm{P}<0.001)$. Table (2)

\begin{tabular}{|c|c|c|c|c|c|c|c|}
\hline \multirow{3}{*}{ 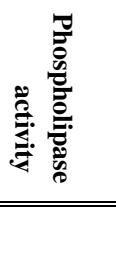 } & \multicolumn{6}{|c|}{ Candida Species } & \\
\hline & \multicolumn{2}{|c|}{$\begin{array}{r}\text { C. albican } \\
(\mathrm{n}=13)\end{array}$} & \multicolumn{2}{|c|}{$\begin{array}{l}\text { C. tropicalis } \\
(\mathrm{n}=27)\end{array}$} & \multicolumn{2}{|c|}{$\begin{array}{r}\text { C. glabrata } \\
(\mathrm{n}=10)\end{array}$} & P-value \\
\hline & $\mathrm{N}$ & $\%$ & $\mathrm{~N}$ & $\%$ & $\mathrm{~N}$ & $\%$ & \\
\hline Negative & 0 & 0.0 & 12 & 44.4 & 10 & 100 & $<0.001^{* *}$ \\
\hline+ & 0 & 0.0 & 15 & 55.6 & 0 & 0 & $<0.001^{* *}$ \\
\hline++ & 2 & 15.4 & 0 & 0.0 & 0 & 0 & $0.052^{*}$ \\
\hline Total & 13 & 100 & 27 & 100 & 10 & 100 & \\
\hline
\end{tabular}

Table 2: Phospholipase activity of different Candida species

Proteinase activity: among the 50 Candida isolates, $34(68.0 \%)$ were positive proteinase. The highest enzymatic activity was reported for C. albicans isolates as $84.6 \%(11 / 13)$ showed strong $(+++)$ proteinase activity, $7.7 \%(1 / 13)$ showed moderate enzyme activity $(++)$ and $7.7 \%(1 / 13)$ showed weak enzymatic activity $(+)$. While, C. tropicalis showed that $63.0 \%(17 / 27)$ showed only weak (+) enzymatic activity and $37.0 \%(10 / 27)$ were negative (-) However, C. glabrata was the lowest proteinase producer as $40 \%(4 / 10)$ showed weak (+) enzymatic activity and $60.0 \%(6 / 10)$ were negative (-). The difference was statistically highly significant $(\mathrm{P}<0.001)$, Table (3) 


\begin{tabular}{|c|c|c|c|c|c|c|c|}
\hline \multirow{3}{*}{ 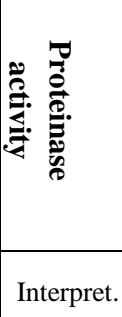 } & \multicolumn{7}{|c|}{ Candida Species } \\
\hline & \multicolumn{2}{|c|}{$\begin{array}{c}\text { C. albican } \\
(n=13)\end{array}$} & \multicolumn{2}{|c|}{$\begin{array}{c}\text { C. } \\
\text { tropicalis } \\
(\mathbf{n}=27)\end{array}$} & \multicolumn{2}{|c|}{$\begin{array}{c}\text { C. } \\
\text { Glabrata } \\
(n=10)\end{array}$} & P-value \\
\hline & $\mathrm{N}$ & $\%$ & $\mathrm{~N}$ & $\%$ & $\mathrm{~N}$ & $\%$ & \\
\hline Negative & 0 & 0.0 & 10 & 37.0 & 6 & 60 & $0.007^{*}$ \\
\hline+ & 1 & 7.7 & 17 & 63.0 & 4 & 40 & $0.004 *$ \\
\hline++ & 1 & 7.7 & 0 & 0.0 & 0 & 0 & 0.234 \\
\hline+++ & 11 & 84.6 & 0 & 0.0 & 0 & 0 & $<0.001^{* *}$ \\
\hline Total & 13 & 100 & 27 & 100 & 10 & 100 & \\
\hline
\end{tabular}

*Non-significant **highly significant

Table 3: Proteinase activity of different Candida species

Haemolytic activity: C. albican produce $100 \% \beta$ haemolytic activity, While $\mathrm{C}$. tropicalis produce $77.8 \%, 22.2 \%, \alpha$ and $\beta$ haemolysis respectively, but C. glabrata produce $100 \% \alpha$ haemolysis with highly statistical significant difference $(\mathrm{P}<0.001)$. Table (4)

\begin{tabular}{|c|c|c|c|c|c|c|}
\hline \multirow{3}{*}{ 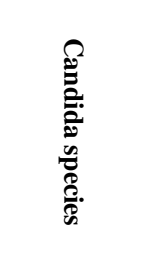 } & \multirow{3}{*}{ 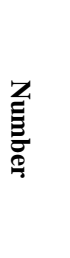 } & \multicolumn{5}{|c|}{ Haemolytic activity } \\
\hline & & \multicolumn{2}{|c|}{ Beta } & \multicolumn{2}{|c|}{ Alpha } & \multirow{2}{*}{ P-value } \\
\hline & & $\mathrm{N}$ & $\%$ & $\mathrm{~N}$ & $\%$ & \\
\hline C. albican & 13 & 13 & 100 & 0 & 0 & $<0.001^{* *}$ \\
\hline C. tropicalis & 27 & 6 & 22.2 & 21 & 77.8 & $<0.001 * *$ \\
\hline C. glabrata & 10 & 0 & 0 & 10 & 100 & $<0.001^{* *}$ \\
\hline
\end{tabular}

** P value highly significant

Table 4: Haemolytic activity of different Candida species

Biofilm formation: C. tropicalis were the highest biofilm producers 27/27 (100\%), 21/27 (77.7\%) were strong producer $(+++)$ and 6/27 (22.2\%) were moderate producer $(++)$ versus $1 / 13 \quad(7.6 \%)$ of C. albicans was strong biofilm producer and $1 / 13(7.6 \%)$ was moderate biofilm producer and the $9 / 13(69.2 \%)$ were weak biofilm producer. However, 9/10 (90\%) of C. glabrata were weak biofilm producer $(+)$ and 1/10 (10\%) was negative biofilm producer $(-)$ with highly statistical significant difference $(\mathrm{P}<0.001)$. Table (5).

\begin{tabular}{|c|c|c|c|c|c|c|c|c|c|}
\hline \multirow{3}{*}{ 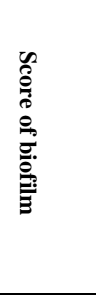 } & \multicolumn{9}{|c|}{ Candida Species } \\
\hline & \multirow{2}{*}{$\begin{array}{c}\mathrm{C} . \\
\text { albican } \\
(\mathrm{N}=13) \\
\mathrm{N}\end{array}$} & \multicolumn{2}{|c|}{$\begin{array}{c}\mathrm{C} . \\
\text { tropicalis } \\
(\mathrm{N}=27)\end{array}$} & \multicolumn{2}{|c|}{$\begin{array}{l}\text { C. glabrata } \\
\qquad(\mathrm{N}=10)\end{array}$} & \multicolumn{3}{|c|}{$\begin{array}{r}\text { Total } \\
(\mathrm{N}=50)\end{array}$} & \multirow[t]{2}{*}{ P-value } \\
\hline & & $\%$ & $\mathrm{~N}$ & $\%$ & $\mathrm{~N}$ & $\%$ & $\mathrm{~N}$ & $\%$ & \\
\hline Negative & 2 & 15.4 & 0 & 0 & 1 & 10 & 11 & 22 & 0.133 \\
\hline+ & 9 & 69.2 & 0 & 0 & 9 & 90 & 18 & 36 & $<0.001^{* *}$ \\
\hline++ & 1 & 7.7 & 6 & 22.2 & 0 & 0 & 7 & 14 & 0.157 \\
\hline+++ & 1 & 7.69 & 21 & 77.8 & 0 & 0 & 22 & 44 & $<0.001^{* *}$ \\
\hline
\end{tabular}

** $\mathrm{P}$ value highly significant

Table 5: Biofilm formation by isolated Candida species

Antifungal susceptibility: Sensitivity of C. albicans was $100 \%$ to Amphotericin and Flucytosine, $76.9 \%$ to Nistatin and Voriconazole, 69.2\% to Ketoconazole and fluconazole, $61.5 \%$ to Miconazole and Itraconazole, $53.8 \%$ to Econazole, and $46.2 \%$ to Clotrimazole. Sensitivity of NAC was $27.0 \%$ to Amphotericin, 94.5\% to Flucytosine, 9.2\% to Nistatin, $10.8 \%$ to Voriconazole and Itraconazole, $75.6 \%$ to Ketonazole, $13.5 \%$ to fluconazole, $8.1 \%$ to Miconazole and $2.7 \%$ to Econazole, and $5.4 \%$ to Clotrimazole, Figure (9).

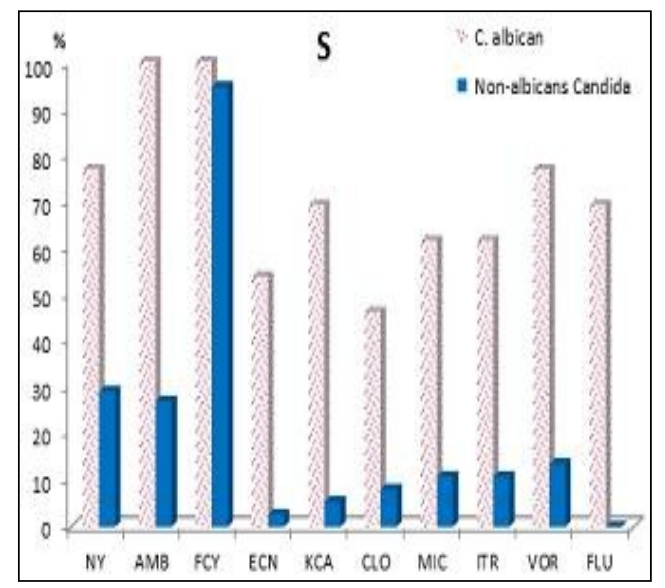

Fig 9: Antifungal susceptibility pattern of the isolated C. albicans versus NAC.

While, C. albicans didn't show any antifungal resistance, most of NAC spp. showed higher rates of resistance to several azole drugs (Clotrimazole $77.6 \%$, Miconazole 70.3\%, Econazole 63.2\%, Nystatine 44.3\%), Figure (10). 


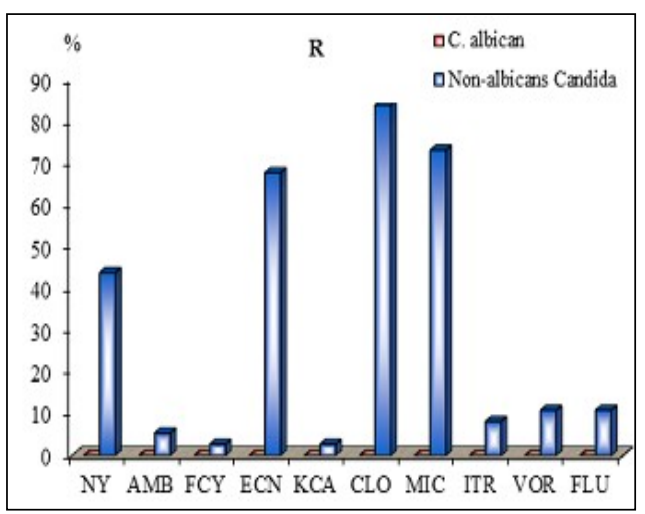

Fig 10 : Antifungal resistance pattern of C. albicans versus NAC.

\section{DISCUSSION}

Catheter associated urinary tract infection (CAUTI) is one of the most common devices associated nosocomial infection. Indwelling urinary catheter is the most important risk factor contributing to the overgrowth of Candida spp. on which Candida can colonize and form biofilm. Similar to other reports, most of patients in our study who confirmed with positive candiduria were between ages $50-70$ years with predominance of female gender $64.0 \%$ (32/50). Jiménez-Guerra et al., $2018^{19}$ found that the average age of patients with candiduria was 64 years. S imilar findings were reported by Melges et al., 2019 [18] who reported that the average age of patients with candiduria was 63 years and $57.0 \%$ were females.

Regarding the associated predisposing risk factors, antibiotics therapy is one of the most important risk factors exposing the patients to candiduria. This correlates with our results as all candiduria positive patients with Urinary catheterization $100 \%$ and $78.0 \%$ (39/50) were on antibiotic treatment. Our results are supported by Melges et al., $2019^{20}$ who reported that antibiotic therapy $89.2 \%$ and the use of an indwelling urinary catheter were $100 \%$ the most frequent predisposing factors. And Chandak et al., $2018^{21}$ who noticed that antibiotics were the most prominent associated risk factor $100 \%$. Diabetes mellitus is a common disease also predisposes to Candida colonization. Our findings showed that $50.0 \%(25 / 50)$ of candiduria positive patients were diabetics in accordance with the results of Melges et al., $2019^{18}$ who reported that $34.4 \%$ of UTI patient with candiduria. Also, Chandak et al., $2018^{21}$ who found that $28.0 \%$ of candiduria positive ICU patients were diabetics.

Our findings showed that $22.8 \%$ (50/219) of catheterized ICU patients had candiduria and this was supported by the results of Montes et al., $2019^{22}$ reported that $37.7 \%$ of Candida spp. isolated from UTI Sahai \& Kumar., $2018^{23}$ who found that out of 100 patients, 26 patients were diagnosed to develop symptomatic CAUTI during their course of hospitalization.

Worldwide, NAC spp. which appear to be better adapted to the urinary tract environment have now replaced C. albicans as the predominant pathogen in nosocomial urinary tract infections Biagi et al., $2019^{24}$

The current study revealed that $74.0 \%$ (37/50) of Candida isolates were NAC species, while $26.0 \%$ (13/50) confirmed as C. albicans. Chandak et al., $2018^{19}$ in their study found that NAC spp. were predominantly high $74.0 \%$, while C. albicans was $26.0 \%$. And Sahai \& Kumar., $2018^{23}$ who reported that NAC spp. contributed to $83.3 \%$ of the isolates and only $16.7 \%$ of isolates were C. albicans. However, in contrary, García-Agudo et al., 2018 ${ }^{25}$ reported that $\mathrm{C}$. albicans was the predominant Candida species $61.9 \%$.

Moreover, our results showed that the NAC species were predominantly isolated as C. tropicalis represented $54.0 \%(27 / 50)$ followed by C. glabrata $20.0 \%(10 / 50)$ and this was in agreement with Melges et al., $2019^{20}$ who reported that C. tropicalis was found most commonly $37.6 \%$, followed by C. albicans $36.6 \%$ and C. glabrata $19.3 \%$. And Chandak et al., $2018^{21}$ who showed that C. tropicalis was the most common amongst NAC representing $72.9 \%$ followed by C. glabrata 16. 3\%. However, in contrary, Montes et al., 2019 22 reported that the most frequent species were C. albicans 42.9\%, C. tropicalis $20.9 \%$ and C. glabrata $16.9 \%$.

In the present work, all recovered Candida spp. were assessed for the production of extracellular enzymes as virulence factors including phospholipase, proteinase and haemolysin.

According to our results, C. albicans had the highest phospholipase activity as $84.6 \%(11 / 13)$ of the C. albicans isolates had strong phospholipase activity and $15.4 \%(2 / 13)$ had moderate enzymatic activity. While $44.4 \%(12 / 27)$ of $C$. tropicalis had no phospholipase (-), 55.6\% (15/27) had weak enzymatic activity and all isolates of C. glabrata had no enzymatic activity at all. Similar results were reported by Dabiri, et al., $2018^{26}$ who founded that the highest positivity of phospholipase production was related to C. albicans $56.8 \%$ followed by C. tropicalis $50.0 \%$ and C. glabrata isolates $20.0 \%$.

However, in case of proteinase enzyme activity it was shown that $\mathrm{C}$. glabrata was the lowest proteinase producer as $40.0 \%(4 / 10)$ showed only weak enzymatic activity and $60.0 \%(6 / 10)$ were negative followed by C. tropicalisas $63.0 \%$ (17/27) showed weak enzymatic activity and $37.0 \%(10 / 27)$ were negative. However the highest enzymatic activity were reported for C. albicans isolates as $84.6 \%$ (11/13) showed strong enzymatic activity, 7.7\% (1/13) showed moderate activity and 7.7\% (1/13) showed weak activity with highly statistical significant differences $(\mathrm{P}<0.001)$, in agreement with the results of Dabiri et al., $2018^{26}$ who reported that C. albicans showed the highest proteinase and phospholipase activity and biofilm formation ability. And Alenzi, $2016^{27}$ founded that Secretory aspartic proteinase activity was demonstrated in $48.0 \%$ of Candida isolates.

Meanwhile, haemolysin secretion followed by iron acquisition facilitates deeper tissue invasion by Candida, Tsang et al., $2007^{28}$. In the present study C. 
albicans produce beta haemolytic activity, this in agreement with Zarrin et al., $2015^{29}$ who showed that all C. albicans isolates exhibited heamolytic activity. All C. tropicalis were haemolysin producer alpha and beta. This in agreement with Udayalax \& Shenoy., $2016^{30}$ who reported that all isolates of Candida spp. both C. albicans and NAC species, produced haemolysin (100\%). While Sachin et al., 2012 found only $30.4 \%$ of C. tropicalis showed haemolytic activity.

One of the main virulence factors are surface molecules that permit adherence of the organism to the structures (e.g., human cells, extra cellular matrix, and prosthetic devices). A biofilm is a community of microorganisms and their extra cellular polymers that are attached to a surface Shekar \& Kabbin., 2015 2 .

Out of the 50 Candida isolates 22 (44.0\%) isolates showed strong biofilm formation, 7/50 (14.0\%) strains showed moderate biofilm formation and 18/50 (36.0\%) strains showed weak biofilm formation. The results were comparable with the standard ATCC strains.

Biofilm production was reported in our study mostly to NAC species $97.3 \%$ (36/37) than C. albicans $84.6 \%(11 / 13)$ and this is also in accordance with Yarlagadda et al., $2017^{33}$ who showed that biofilm production was detected most frequently among NAC species $48.0 \%$ than C. albicans $25.0 \%$. As well as Sida et al., $2016^{34}$ reported that biofilm production was found to occur by $65.2 \%$ of NAC species compared to $34.7 \%$ of C. albicans. Also, Shekar \& Kabbin., $2015^{30}$ reported that out of the 26 Candida isolates 14 (53.8\%) were biofilm producer, 10/14 (71.5\%) were NAC while 4/14 (28.5\%) only were related to C. albicans. However, Al kilani et al., $2017^{35}$ found that biofilm formation is more in C. albicans versus C. tropicalis $83.3 \%$ and $57.9 \%$, respectively.

The major strong biofilm producer was $\mathrm{C}$. tropicalis $77.7 \%$ (21/27) followed by C. albicans 7.7\% (1/13) with highly significant difference $(\mathrm{P}<0.001)$. Biofilm formation also was reported in $63.6 \%$ of $\mathrm{C}$. tropicalis versus $(21.2 \%)$ for C. albicans in a study done by Chandak et al., $2018^{21}$

Several studies have reported that amphotericin B was the most effective drug against Candida isolates. Fluconazole is considered as a potentially useful drug in Candida UTI due to its high concentration in urine and better tolerance; however, increasing resistance to azoles is being reported.

In our study, the results showed that C. albicans was sensitive to all tested antimycotic drugs with maximum sensitivity $100 \%$ to Flucytosine and Amphotericin and the other night antimycotic showed either sensitivity or intermediate sensitivity without absolute resistance. In concordance with this finding, Sahai \& Kumar., $2018^{23}$ reported that all Candida isolates were sensitive to fluconazole, voriconazole, amphotericin B and itraconazole.

While NAC spp. were sensitive only to Flucytosine $94.59 \%$ and Ketonazole $75.67 \%$, and it was highly resistant to other tested Azole including Clotrimazole
77.6\%, Miconazole 70.3\%, Econazole 63.2\% and Nystatine $44.3 \%$. Similar rates of fluconazole resistance were reported by Yarlagadda et al., $2017^{33}$.

\section{CONCLUSION}

This study documents the prevalence of candiduria in catheterized patients and the change in trend with shift toward non albicans Candida species as the predominant pathogen causing nosocomial UTI. A wide range of intra- and inter- species enzymatic activities and biofilm forming ability have been accounted for testing the pathogenicity of Candida species in susceptible individuals, while correlation with clinical outcomes is still need to be validated. Biofilm formation is seen more frequently with non albicans candida species than with Candida albicans and as biofilm production may help maintain the role of fungi as commensals and pathogen, by evading host defense mechanisms, resisting fungal treatment and withstanding the competitive pressure from other organisms, these are difficult to treat. Studies of urine cultures in hospitalized patients should include the possible presence of candiduria. Species identification and biofilm detection must be performed for early and effective treatment of the patient.

\section{REFERENCES}

1. Gajdács M., Dóczi I., Ábrók M., Lázár A. and Burián K.: Epidemiology of candiduria and Candida urinary tract infections in inpatients and outpatients: results from a 10-year retrospective survey. Cent. European J. Urol., 2019; 72(2), 209-14.

2. Frías-De-León M.G., Martínez-Herrera E., Acosta-Altamirano G., Arenas R., and Rodríguez-Cerdeira C.: Superficial candidosis by Candida duobushaemulonii: An emerging microorganism. Infect. Genet. Evol. 2019, 75, 103960.

3. Fazeli, A.; Kordbacheh, P.; Nazari, A.; Daie Ghazvini, R.; Mirhendi, H.; Safara, M.; Bakhshi, H.; Yaghoubi, R.: Candiduria in Hospitalized Patients and Identification of Isolated Candida Species by Morphological and Molecular Methods in Ilam, Iran. Iran J. Public Health, 2019; 48, 156-61.

4. Ortiz, B.; Perez-Aleman, E.; Galo, C.; and Fontecha, G.: Molecular identification of Candida species from urinary infections in Honduras. Rev. Iberoam. Micol., 2018; 35, 73-7.

5. Alfouzan, W.; Dhar, R.; Albarrag A.; and AlAbdely, H.: The emerging pathogen Candida auris: A focus on the Middle-Eastern countries. J. Infect. Public Health, 2019; 12, 451-9.

6. Raminder Sandhu, Shalley Dahiya, Pallavi Sayal, and Diksha Budhani: Increased role of non albicans Candida, potential risk factors, and attributable mortality in hospitalized patients. Journal of Health Research and Reviws, 2017; Volume: 4, Issue: 2, Page: 78-83. 
7. El-Houssaini H. H, Elnabawy O. M, Nasser H. A and Elkhatib W. F: Correlation between antifungal resistance and virulence factors in Candida albicans recovered from vaginal specimens. Microb Pathog, 2019; 128: 13-9.

8. Cavalheiro M. and Teixeira M. C.: Candida Biofilms: Threats, Challenges, and Promising Strategies. Front. Med. 2018; 5:28. doi: 10.3389/fmed.00028

9. Kurtzman, C. P., Fell, J. W., and Boekhout, T.: The Yeasts Taxonomic Study $5^{\text {Th }}$ (edn.) Jamestown, London-United Kingdom, 2011; Elsevier Science Publishers.

10. Gharanfoli A., Mahmoudi E., Torabizadeh R., Katiraee F. and Faraji S.: Isolation, characterization and molecular identification of Candida species from urinary tract infections. Curr. Med. Mycol, 2019; 5(2), 33-6.

11. Calderone E., Richard A. and Cornelius J. Clancy: Candida and Candidiasis, Second Edition. Fungi and Fungal Pathogenesis, 2002; Clinical Microbiology.

12. Albaina, O., Sahand, I. H., Brusca, M. I., Sullivan, D. J., Fernandez de Larrinoa, I. and Moragues, M. D.: Identification and characterisation of nine atypical Candida dubliniensis. Clinical isolates. Journal of Medical Microbiology, 2015; 64, 147-156.

13. Price M. F., Wilkinson I. D. and Gentry L. O.: Plate method for detection of phospholipase activity in Candida albicans. Sabouraudia, 1982; 20:7-14.

14. Chakrabarti A, Nayak N, Talwar P. In vitro proteinase production by Candida species. Mycopathologia 1991; 114:163-8

15. Patil S, Ugargol AR, Srikanth NS. Comparison of two methods or detection of secreted aspartyl proteinase in urinary isolates of candida species. National Journal of medical research 2014; 4: 119-21

16. Manns J. M., Mosser D. M. and Buckley H. R.: Production of a hemolytic factor by Candida albicans. Infection and Immunity, 1994; 62 (11): 5154-6.

17. Yigit N., Aktas E., Dagistan S., and Ayyildiz A.: Investigating Biofilm Production, Coagulase and Hemolytic Activity in Candida Species Isolated from Denture Stomatitis Patients from denture stomatitis patients. Eurasian $\mathrm{J}$ Med, 2011; 43: 27-32.

18. Freeman D. J., Falkiner F. R. and Keane C. T.: New method for detecting slime production by coagulase negative Staphylococci. J. Clin Pathol. 1989; 42:872-4.

19. Jiménez-Guerra G, Casanovas Moreno-Torres I, Gutiérrez-Soto $\mathrm{M}$, Vazquez-Alonso $\mathrm{F}$ and Sorlózano-Puerto A, et al.: Inpatient candiduria: etiology, susceptibility to antifungal drugs and risk factors Inpatient candiduria: etiology, susceptibility to antifungal drugs and risk factors. Rev Esp Quimioter, 2018; 31(4): 323-8.

20. Melges M. Pesenti de Santana, Hugo D. Hoffmann-Santos, Luciana Basili D. Tomoko Tadano, et al.: Epidemiological profile of patients hospitalized with candiduria in the Central-Western region of Brazil. Rev beroam Micol, 2019; 36(4):175-80.

21. Chandak R. J., Bibhabati Mishra, Archana Thakur, Poonam Sood Loomba, and Vinita Dogra: Comparison of Methods of Biofilm Detection in Urinary Candida Isolates and Evaluating its Role in Persistent Candiduria. National Journal of Laboratory Medicine, 2018; Vol-7(2): MO06-MO11

22. Montes K., Bryan Ortiz, Celeste Galindo, Isis Figueroa, Sharleen Braham and Gustavo Fontech: Identification of Candida Species from Clinical Samples in a Honduran Tertiary Hospital. Pathogens, 2019; 8, 237; doi: 10.3390/pathogens8040237.

23. Sahai S. \& Kumar A.: Role of Candida in Catheter Associated Urinary Tract Infection. Int J Cur Res Rev., 2018; Vol 10, Issue 20, 15-9.

24. Biagi M. J., Wiederhold N. P., Gibas C. et al.: Development of high-level echinocandin resistance in a patient with recurrent Candida auris candidemia secondary to chronic candiduria. Open Forum Infect. Dis., 2019; 6(7), ofz262.

25. García-Agudo L., Manuel Rodríguez-Iglesias \& Rafael Carranza-González (2018): Nosocomial Candiduria in the Elderly: Microbiological Diagnosis. Mycopathologia volume 183, pages 591-6.

26. Dabiria S.M. Shams, Ghahfarokhia M. and Razzaghi-Abyanehb: Comparative analysis of proteinase, phospholipase, hydrophobicity and biofilm forming ability in Candida species isolated from clinical specimens. https://doi.org/10.1016/j.mycmed.2018.04.00 , Vol. 28(3), Pages 437-442.

27. Alenzi F. Q. B.: Virulence factors of Candida species isolated from patients with urinary tract infection and obstructive uropathy. Pak J Med Sci, 2016; Vol. 32 No. 1 www.pjms.com.pk.

28. Tsang C.S. P, Chu F. C. S, Leung W. K, Jin L. J, Samaranayake LP and Siu S. C.: Phospholipase, proteinase and haemolytic activities of Candida albicans isolated from oral cavities of patients with type 2 diabetes mellitus. J. Med Microbiol., 2007; 56(Pt 10):1393-1398. doi: 10.1099/jmm.0.47303-0.

29. Zarrin M., Mahsa Rajabi, Ali Zarei and Mahmoud Abadi: Study of haemolytic, esterase activities and germ tube formation in Candida albicans from patients with vaginitis and urinary infections. Brunei Int Med J. 2015; 11 (4): 191-6. 
30. Udayalax and Neetha Shenoy: Comparison Between Biofilm Production, Phospholipase and Haemolytic Activity of Different Species of Candida Isolated from Dental Caries Lesions in Children. J Clin Diagn Res. 2016 Apr; 10(4): DC21-DC23.

31. Sachin C.D, Ruchi K, and Santosh S.: In vitro evaluation of proteinase, phospholipase and haemolysin activities of Candida species isolated from clinical specimens. Int J Med Biomed Res, 2012; 1(2):153-7.

32. Shekar M. R. and Kabbin J. S.: Candiduria in Catheter Associated Urinary Tract Infection with Special Reference to Biofilm Production. J Clin Diagn Res., 2015; 9(10): DC11-DC13.
33. P. Yarlagadda, Uma Penmetcha, Naseema Shaik and Ramesh Babu Myneni : Study of Biofilm Formation \& Drug Resistance Pattern in Various Candida Species Isolated from Patients with Urinary Tract Infection. International Journal of Current Microbiology and Applied Sciences, 2017; Volume 6, Number 9, pp. 3423-30.

34. Hetal Sida, Parul Shah, Jayshri Pethani, Lata Patel and Hiral Shah: Study of biofilm formation as a virulence marker in Candida species isolated from various clinical specimens. International Journal Medical Science and Public Health, 2016; Vol 5, Issue 05, Page 842-6.

35. Alkilani A. A, El Shalakany A. H, and Mohamed Saif W. Y: Candiduria in catheterized Menoufia patients: emerging microbiological trends. Menoufia Med J, 2017; 30:892-8
Microbiology and Immunology 